\title{
Presumed Alive
}

National Cancer Institute

\section{Source}

National Cancer Institute. Presumed Alive. NCI Thesaurus. Code C141463.

The assumption that a person is still living. 